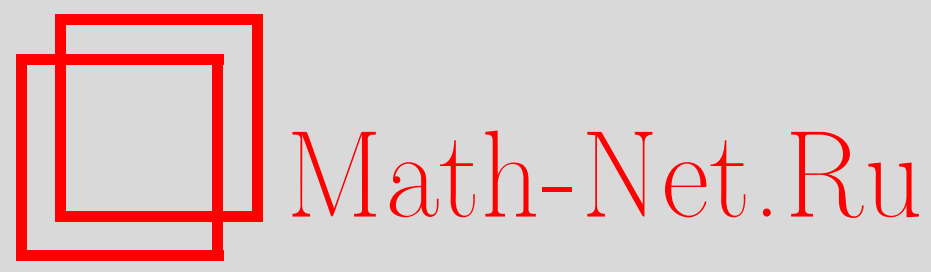

Э. Я. Еленицкий, Краевая задача для гибких осесимметрично нагруженных составных оболочек вращения и стержневых систем, Вестн. Сам. гос. техн. ун-та. Сер. Физ.-мат. науки, 2012, выпуск 4(), 122-130

DOI: https://doi.org/10.14498/vsgtu1191

Использование Общероссийского математического портала Math-Net.Ru подразумевает, что вы прочитали и согласны с пользовательским соглашением

http://www . mathnet.ru/rus/agreement

Параметры загрузки:

IP : 18.234 .197 .8

26 апреля 2023 г., 18:32:21

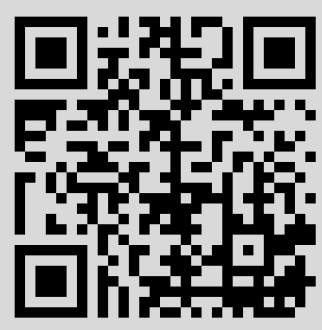


Вестн. Сам. гос. техн. ун-та. Сер. Физ.-мат. науки. 2012. № 4 (29). С. $122-130$

УДК 539.3:517.95:534.1

\title{
КРАЕВАЯ ЗАДАЧА ДЛЯ ГИБКИХ ОСЕСИММЕТРИЧНО НАГРУЖЕННЫХ СОСТАВНЫХ ОБОЛОЧЕК ВРАЩЕНИЯ И СТЕРЖНЕВЫХ СИСТЕМ
}

\author{
Э. Я. Еленицкий \\ Самарский государственный архитектурно-строительный университет, \\ 443001, Россия, Самара, ул. Молодогвардейская, 194. \\ E-mail: elenit@list.ru
}

\begin{abstract}
Предлагается эффективный алгоритм статического расчёта геометрически нелинейных составных тонкостенных конструкиий. Исполъзуются линейные дифференциальные соотношения моментной теории. Нелинейность учитывается путём осуществления заранее не известного начального углового смещения каждого участка, сохраняющего форму образующей. Неизвестными разрешаюшей системы алгебраических уравнений являются произвольные постоянные общего решения и начальные углы поворота образующих. Линеаризация осуществляется итерационным методом Ньютона-Рабсона и обеспечивает высокую точность результатов.
\end{abstract}

Ключевые слова: геометрическая нелинейность, стержень, оболочка вращения, угловое смещение, дифберенциальные уравнения, итерационный расчёт.

Введение. Большинство исследований конечных перемещений гибких конструкций основано на математических моделях, включающих системы нелинейных дифференциальных уравнений $[1,2]$. В настоящей статье предлагается альтернативный подход, позволяющий выполнять расчёты гибких плоских стержневых систем и осесимметрично нагруженных составных оболочек вращения без применения нелинейных дифференциальных зависимостей. Перемещения таких систем соизмеримы с размерами стержня (оболочки), а относительные деформации остаются малыми. Последнее обстоятельство указывает на то, что для каждого участка конструкции целесообразно выделить две группы перемещений, первая из которых соответствует его переносу без изменения формы образующей (нелинейная часть), а вторая - изгибу и продольному растяжению - сжатию (линейная часть). Первая группа перемещений определяется тремя степенями свободы: двумя линейными перемещениями и углом поворота начального сечения участка, что значительно упрощает последующий анализ напряжённо-деформированного состояния. Вторая группа перемещений и соответствующих им внутренних усилий описывается классическими линейными дифференциальными уравнениями и их интегралами [3]. Поскольку перемещения второй группы являются бесконечно малыми, возможна суперпозиция решений обоих этапов движения. Кинематические и статические условия сопряжения и внешнего закрепления границ участков обеспечивают формирование нелинейной системы алгебраических уравнений. При этом неизвестными задачи являются угловые смещения переносного движения, а также $6 n$ ( $n$ - число участков) постоянных интегрирования общих решений дифференциальных уравнений. Каждый шаг построенного итерационного процесса включает решение линейной краевой задачи

Эдуард Яшевич Еленицкий (к.т.н., доц.), доцент, каф. сопротивления материалов и строительной механики. 
для составной конструкции при угловых смещениях её участков, полученных на предыдущей итерации, и уточнения величины угловых смещений следующего приближения. Сходимость нелинейного решения обусловлена тем, что процедуры линеаризации имеют простой геометрический смысл, и тем, что используются апробированные замкнутые линейные решения краевых задач. Ввиду того, что неизвестными задачи являются произвольные постоянные, отпадает необходимость построения матрицы жёсткости (податливости), как это предусмотрено в методе перемещений (сил) [4].

1. Построение решения. Рассмотрим элемент, вырезанный из системы, подверженной действию статических нагрузок. Будем относить понятие «элемент» к прямолинейному стержню на линейно упругом основании в условиях плоской деформации, а также к круговым коническим, цилиндрическим оболочкам или пластине при осесимметричном воздействии. Полагая перемещения большими, а деформации малыми, поставим задачу определения положения упругого элемента $n k$ с учётом конечных перемещений, вызванных действием следящих внеузловых нагрузок $\mathbf{p}(x)$, и условий закрепления на краях, допускающих действие силовых и кинематических факторов (рис. 1). При этом исходное положение элемента $n_{0} k_{0}$ длиной $L$ в фиксированной глобальной системе отсчета $X Z$ определяется декартовыми координатами и углом наклона $\alpha$ начального сечения. Прямая $O Z$ является осью вращения, а отрезок $n_{0} k_{0}$ - образующей пластины $(\alpha=0)$, цилиндрической $(\alpha=\pi / 2)$ или конической $(0<\alpha<\pi / 2)$ оболочки. Для стержня его исходное положение также совпадает с отрезком $n_{0} k_{0}$. Расчётное сечение элемента имеет координату $x$, для которой

$$
x=x_{0}+\Delta x, \quad x_{0} \leqslant x \leqslant L,
$$

где для стержня и цилиндрической оболочки координата начального сечения $x_{0}=0$, для круглой пластины и конической оболочки $x_{0}=O_{1} n_{0}$.

В рамках разработанного метода полагаем, что деформирование элемента происходит в два этапа: до некоторого положения $n_{0} k_{i}$ и затем - до конечного положения $n k$. При этом угловое смещение на первом этапе не приводит к изменению формы образующей и определяется углом поворота $\varphi_{i}$, получаемым в процессе расчёта таким образом, чтобы отрезки $n_{0} k_{i}$ и $n k$ оказались параллельными. Указанное смещение реализуется приложением к элементу трёхкомпонентного вектора дополнительных нагрузок $\mathbf{p}_{r}(x)$, имеющего такую же структуру, что и вектор $\mathbf{p}(x)$. Здесь и в дальнейшем индекс $r$ означает поворот - «rotation». Продольные и моментные нагрузки на рис. 1 условно не показаны.

Поскольку движение $n_{0} k_{0}-n_{0} k_{i}$ сопровождается нелинейными перемещениями, используем систему отсчета $X_{i} Z_{i}$, которая оказывается в конечном положении первой стадии деформирования. Введем подлежащие определению вектор-функции перемещений $\mathbf{d}_{i}$ и усилий $\mathbf{f}_{i}$ :

$$
\mathbf{d}_{i}(x)=\left[\begin{array}{lll}
u_{i}(x) & w_{i}(x) & \psi_{i}(x)
\end{array}\right]^{\top}, \quad \mathbf{f}_{i}(x)=\left[\begin{array}{lll}
N_{i}(x) & Q_{i}(x) & M_{i}(x)
\end{array}\right]^{\top},
$$

зависящие от выбранной системы координат следующим образом:

$$
\mathbf{d}_{0}=h\left(\varphi_{i}\right) \mathbf{d}_{i}, \quad \mathbf{f}_{0}=h\left(\varphi_{i}\right) \mathbf{f}_{i}, \quad h\left(\varphi_{i}\right)=\left[\begin{array}{ccc}
\cos \varphi_{i} & -\sin \varphi_{i} & 0 \\
\sin \varphi_{i} & \cos \varphi_{i} & 0 \\
0 & 0 & 1
\end{array}\right]
$$




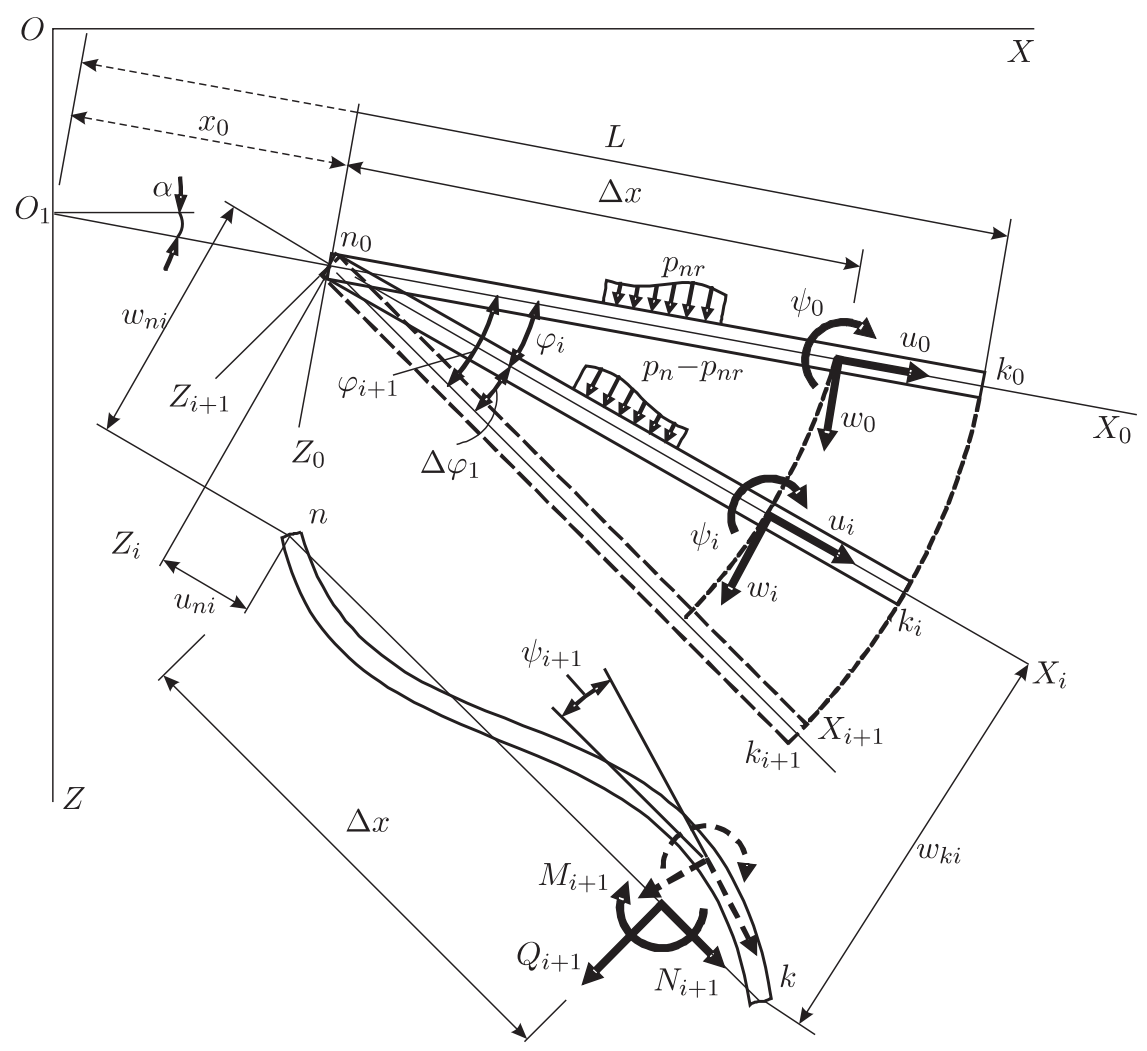

Рис. 1. Схема нелинейного деформирования элемента

Полагаем, что линейная часть соотношений (1) удовлетворяет решению дифференциальных уравнений равновесия моментной теории Кирхгофа-Лява:

$$
L \mathbf{d}_{i}(x)=\mathbf{p}(x), \quad \mathbf{f}_{i}(x)=L_{f} \mathbf{d}_{i}(x) .
$$

В соотношениях (1)-(3) $u_{i}, w_{i}, \psi_{i}$ - продольные, поперечные и угловые перемещения расчётного сечения; $N_{i}, Q_{i}, M_{i}$ - соответствующие меридиональные усилия растяжения, поперечного сдвига и изгиба; $h\left(\varphi_{i}\right)$ - матрица преобразования векторов из подвижной $X_{i} Z_{i}$ в фиксированную $X_{0} Z_{0}(i=$ $=0)$ систему координат; $L, L_{f}$-матрицы дифференциальных операторов соответственно для уравнений равновесия и физических соотношений между усилиями и перемещениями. Структура этих матриц имеет следующий вид [3]:

$$
L=\left[\begin{array}{ccc}
L_{11} & L_{12} & 0 \\
L_{21} & L_{22} & 0 \\
0 & 0 & 0
\end{array}\right], \quad L_{f}=\left[\begin{array}{ccc}
L_{f 11} & L_{f 12} & 0 \\
0 & L_{f 22} & 0 \\
0 & L_{f 23} & 0
\end{array}\right]
$$

Движение элемента из исходного $n_{0} k_{0}$ в конечное положение $n k$ характеризуется перемещением расчётного сечения по траектории $m_{0} m_{2} m_{3} m_{4}$ (рис. 2). Заменяем перемещение по дуге $m_{0} m_{2}$ движением вдоль отрезков $m_{0} m_{1}, m_{1} m_{2}$, параллельных осям $Z_{i}, X_{i}$. При этом обжатие участка $n_{0} m_{0}$ при его переходе в положение $n_{0} m_{1}$ компенсируется растяжением при последующем движении 


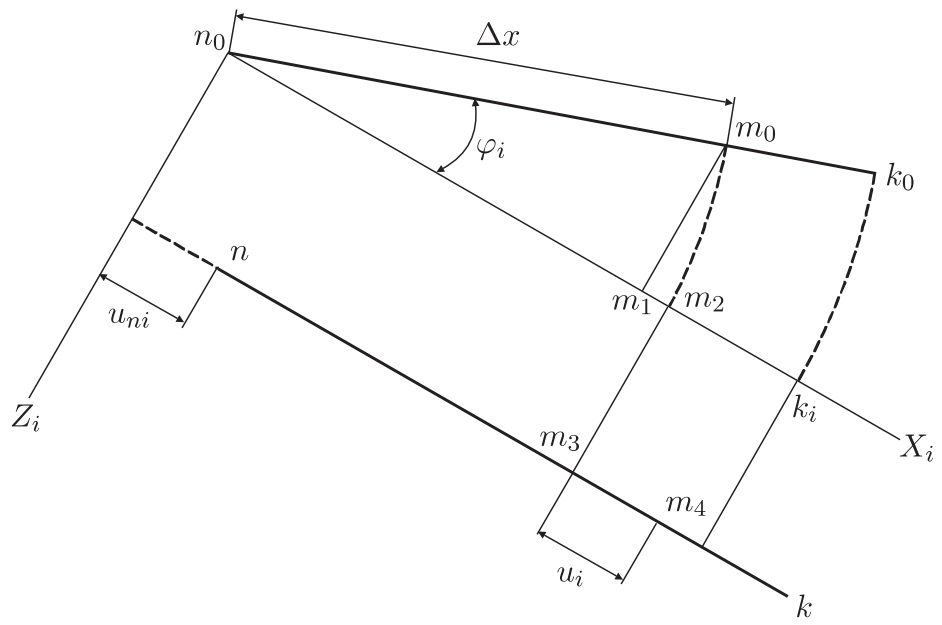

Рис. 2. Траектория перемещения фиксированного сечения

до положения $n_{0} m_{2}$. В случае линейного решения перемещение точки $m_{0}$ по траектории $m_{0} m_{1}$ не сопровождается обжатием участка $n_{0} m_{0}$. Поэтому будем полагать, что последующее движение расчётного сечения до точки $m_{2}$ происходит без увеличения продольных деформаций элемента, что компенсирует отмеченную выше потерю деформаций сжатия. Таким образом, перемещение расчётного сечения на участке $m_{1} m_{2}$ характеризуется следующими зависимостями:

$$
u_{i}(x)=\Delta x\left(1-\cos \varphi_{i}\right), \quad w_{i}(x)=0, \quad N_{i}(x)=0 .
$$

Полные перемещения $\mathbf{d}_{i}$ в координатах $X_{i} Z_{i}$ определяются векторами

$$
\mathbf{d}_{i}=\mathbf{d}_{e i}+\mathbf{d}_{r i}+\boldsymbol{\Delta}_{d i}
$$

где

$$
\mathbf{d}_{r i}=\left[\begin{array}{lll}
0 & \Delta x \sin \varphi_{i} & \sin \varphi_{i}
\end{array}\right]^{\top}, \Delta_{d i}=\left[\begin{array}{lll}
\Delta x\left(1-\cos \varphi_{i}\right) & 0 & \left(\varphi_{i}-\sin \varphi_{i}\right)
\end{array}\right]^{\top} .
$$

Здесь сумма векторов $\mathbf{d}_{r i}+\boldsymbol{\Delta}_{d i}-$ перемещения по траектории $m_{0} m_{1} m_{2}$ (этап 1), вектор $\mathbf{d}_{e i}-$ перемещения по траектории $m_{2} m_{3} m_{4}$ (этап 2 ). Первые два слагаемых в правой части равенства (6) представляют линейную часть решения, последнее учитывает конечность угла поворота $\varphi_{i}$.

Перемещениям (6) соответствуют усилия, определяемые вторым соотношением (3), то есть

$$
\mathbf{f}_{i}=L_{f}\left(\mathbf{d}_{e i}+\mathbf{d}_{r i}+\boldsymbol{\Delta}_{d i}\right)=\mathbf{f}_{e i}+\mathbf{f}_{r i}+\boldsymbol{\Delta}_{f i}
$$

где с учётом зависимостей (4), (5), (7)

$$
\boldsymbol{\Delta}_{f i}=L_{f}\left(\boldsymbol{\Delta}_{d i}\right)=0 .
$$

Подстановка линейной части соотношений (6) в исходную систему уравнений равновесия (3) преобразует её к виду

$$
L \mathbf{d}_{e i}=\mathbf{p}-\mathbf{p}_{r i}, \quad \mathbf{p}_{r i}=L \mathbf{d}_{r i} .
$$


Представим интегралы уравнений (10) следующим образом:

$$
\mathbf{d}_{e i}=a_{d i} \mathbf{C}_{i}+\mathbf{d}_{p i}-\mathbf{d}_{r i}, \quad \mathbf{f}_{e i}=a_{f i} \mathbf{C}_{i}+\mathbf{f}_{p i}-\mathbf{f}_{r i},
$$

где $a_{d i}$ - матрица размерностью $3 \times 6$ фундаментальных решений для элемента в положении $n_{0} k_{i} ; a_{f i}$ - матрица той же размерности, получаемая с помощью второго равенства (3); $\mathbf{C}_{i}$ - шестикомпонентный вектор-столбец произвольных постоянных общего решения; $\mathbf{d}_{p i}, \mathbf{d}_{r i}, \mathbf{f}_{p i}, \mathbf{f}_{r i}$ - частные интегралы, соответствующие нагрузкам $\mathbf{p}, \mathbf{p}_{\text {ri }}$.

Выражения для перемещений (6) и усилий (8) с учётом соотношений (9), (11) принимают окончательный вид:

$$
\mathbf{d}_{i}=a_{d i} \mathbf{C}_{i}+\mathbf{d}_{p i}+\boldsymbol{\Delta}_{d i}, \quad \mathbf{d}_{i}=a_{f i} \mathbf{C}_{i}+\mathbf{f}_{p i}
$$

Дальнейшие действия сводятся к формированию граничных условий и решению системы линейных алгебраических уравнений относительно неизвестных произвольных постоянных. Однако следует иметь в виду, что зависимости $(2),(12)$ справедливы, когда линии $n_{0} k_{i}$ и $n k$ параллельны. Поскольку окончательное положение элемента заранее не известно, то угол $\varphi_{i}$ должен определяться на основе итерационного расчёта. Для очередной $(i+1)$-й итерации имеем (см. рис. 1):

$$
\varphi_{i+1}=\varphi_{i}+\Delta \varphi_{i}, \quad \operatorname{tg} \Delta \varphi_{i}=\left(w_{k i}-w_{n i}\right) / L
$$

где $w_{n i}, w_{k i}$ - нормальные линейные перемещения концевых сечений элемента, полученные на $i$-том шаге расчёта.

Линеаризацию решения проводим методом Ньютона-Рафсона по схеме

$$
\Delta \mathbf{P}_{i}=\mathbf{P}_{i}-A_{i} \mathbf{C}_{i}, \quad \mathbf{C}_{i+1}=\mathbf{C}_{i}+A_{i}^{-1} \Delta \mathbf{P}_{i}
$$

где $A_{i}, \mathbf{P}_{i}, \Delta \mathbf{P}_{i}$ - соответственно невырожденная матрица коэффициентов при неизвестных, столбец свободных членов и невязка разрешающей системы алгебраических уравнений для $i$-той итерации.

Процесс итераций по (13) начинается с линейного расчёта при $\varphi_{i}=0$ и производится до тех пор, пока величина $\Delta \varphi_{i}$ не станет меньше заданной точности. Как известно, метод Ньютона-Рафсона обеспечивает быструю сходимость, а в случае решения задач с весьма сильной нелинейностью успешно сочетается с методом пошагового приращения нагрузки.

Окончательный результат для усилий после обеспечения сходимости итерационного процесса следует представить в виде

$$
\mathbf{f}_{i 0}=h\left(\psi_{i}\right)^{-1} \mathbf{f}_{i}
$$

где квадратная матрица в правой части равенства осуществляет преобразование полученных усилий $\mathbf{f}_{i}$ на криволинейную ось элемента (см. рис. 1).

Поскольку приведённые процедуры основаны на замкнутых решениях дифференциальных уравнений, исключается влияние на результат дополнительных погрешностей, связанных с конечномерными аппроксимациями дифференциальных зависимостей. Если решение (2), (12) получено для элемента, длина которого не обеспечивает малость угла поворота $d w / d x$, следует произвести разбиение исходного интервала на некоторое количество участков и 
сформировать систему алгебраических уравнений с учётом следующих условий сопряжения на внутренних границах участков:

$$
H_{e-1} \mathbf{d}_{0, e-1}\left(L_{e}\right)=H_{e} \mathbf{d}_{0, e}\left(x_{0 e}\right), H_{e-1} \mathbf{f}_{0, e-1}\left(L_{e}\right)=-H_{e} \mathbf{f}_{0, e}\left(x_{0 e}\right)
$$

где $\mathbf{d}_{0, e}, \mathbf{f}_{0, e}$ - матрицы для элемента $e$, имеющие тот же смысл, что и в (2); $x_{0 e}, L_{e}$ - координаты крайних сечений элемента $e ; H_{e}$ - матрица для перехода к глобальной системе координат $X Z ; e-1, e-$ номера смежных элементов.

Замечаем, что наличие матриц $H_{e}$ позволяет также решать задачи для криволинейных стержней и оболочек вращения произвольной формы, образующие которых аппроксимируются ломаной линией с необходимым для заданной точности количеством участков. Кроме того, полученные соотношения допускают применение процедур формирования граничных условий не только для системы последовательно соединенных элементов, но и для составных конструкций ветвящегося типа [5].

2. Тестовые расчёты. Сравним результаты решений некоторых задач нелинейного деформирования гибких конструкций с аналогичными результатами, полученными на основе представленного метода углового смещения.

В качестве первого теста рассмотрим в рамках модели Бернулли консольный стержень постоянного сечения, изгибаемый моментом $M$ (рис. $3, \mathrm{a})$. В линейной постановке кривизна определяется приближенно как вторая производная от функции прогиба, в нелинейной - точно, поскольку зависимость радиуса кривизны $\rho$ от жёсткости стержня $E J$ приводит к следующему результату [6]:

$$
1 / \rho=M /(E J)=\text { const. }
$$

Из геометрических соображений с учётом равенства (14) имеем

$$
u=x-\rho \sin \psi, \quad w=\rho(1-\cos \psi), \quad \psi=x / \rho,
$$

где перемещения $u, w, \psi$ являются, как и ранее, компонентами вектора $\mathbf{d}_{0}$.

Решение задачи на экстремум позволяет определить максимально возможный прогиб на конце стержня $w_{\max }$ и соответствующий этому перемещению момент $M_{0}$ по формулам [6]:

$$
w_{\max }=0,7246 L, \quad M_{0}=2,3311 E J / L \text {. }
$$

При этом максимальный прогиб балки не зависит от её жёсткости, что является весьма удобным для последующего анализа.

На рис. 3, а приведены результаты решения по методике автора. Изображенные на нем перемещения показаны в масштабе размеров стержня, имеющего длину $L=10$ м. Расчёты выполнялись при различном количестве итерационных циклов $i$ с предварительным разбиением стержня на $n=5$ равных участков. Перемещения стержня в линейной постановке $(i=0)$ представлены пунктирной кривой. Результат существенно изменяется после первой итерации $(i=1)$, а при $i=3$ совпадает с точным решением (15), (16).

На рис. 3, б показаны графики зависимости относительной ошибки полученного решения $\varepsilon$ от количества участков разбиения $n$ и количества итераций $i$. Следует отметить, что, несмотря на существенную нелинейность задачи, погрешность результатов не превышает $3 \%$ уже при $n=3, i=2$. 

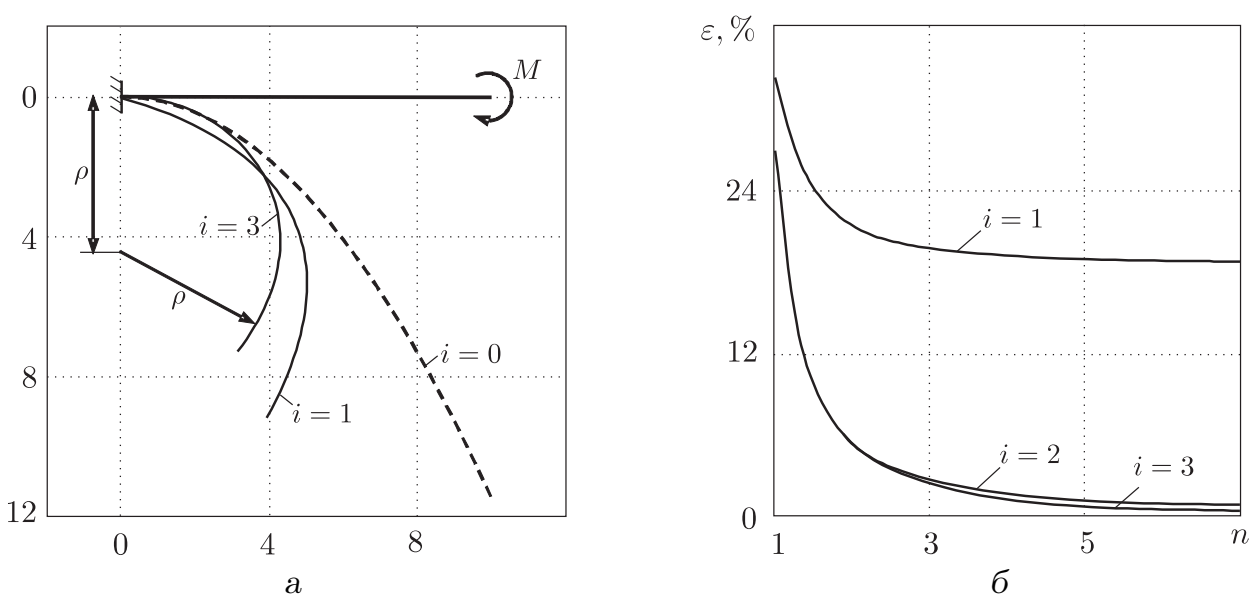

Рис. 3. Изгиб консольного стержня
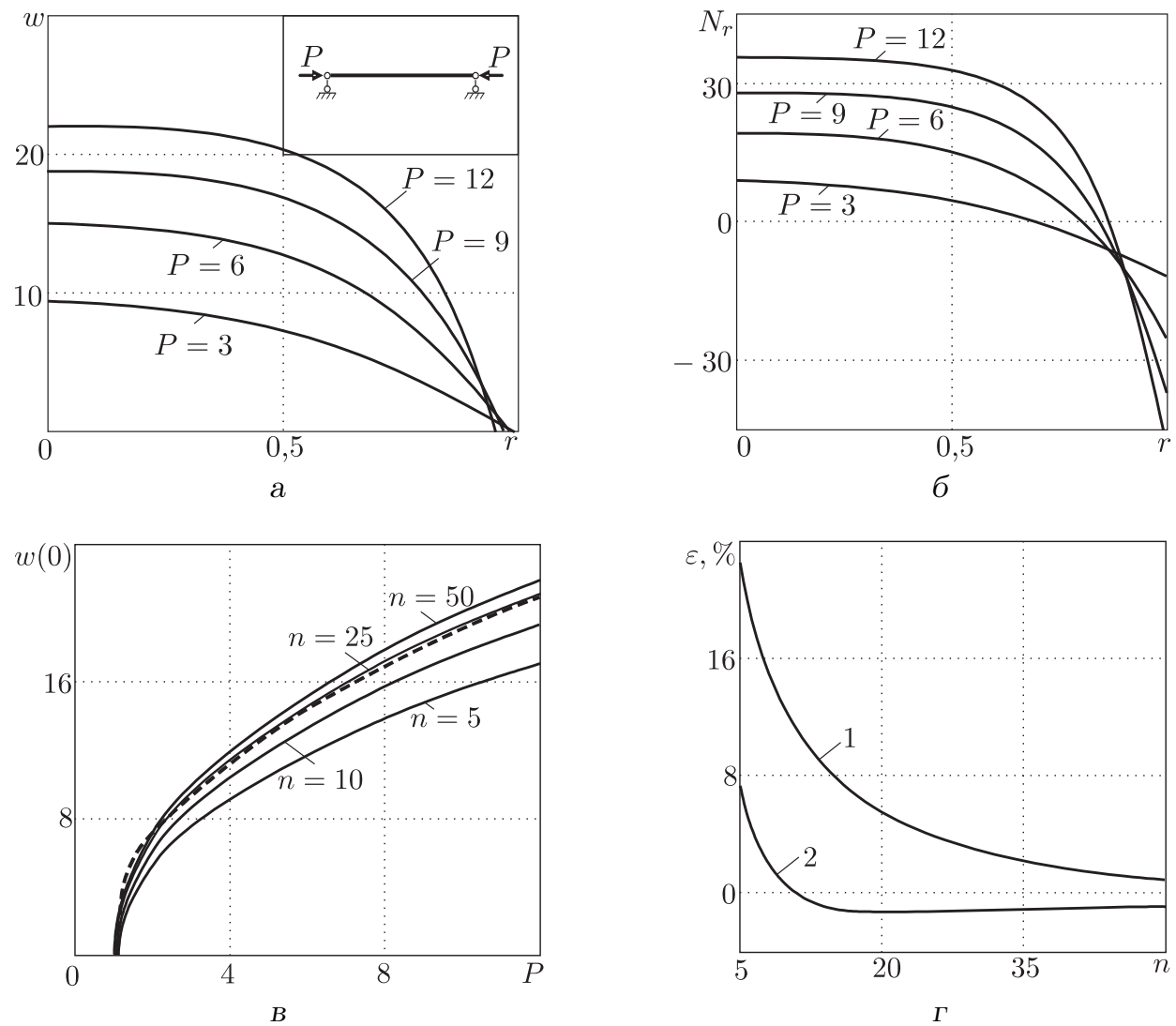

Рис. 4. Закритическое деформирование круглой пластины 
Дальнейшее уточнение решения заданием $n=5, i=2$ снижает относительную ошибку до $1,5 \%$.

В качестве второго теста рассмотрена задача о закритическом поведении шарнирно опертой круглой пластины, находящейся под действием распределенных по контуру сжимающих радиальных усилий $P$ (рис. 4, a). В этом случае пластина способна после потери устойчивости нести возрастающую нагрузку, сохраняя при этом осесимметричную форму деформирования. Стратегия решения основана на том, что на первом шаге к пластине помимо сжимающих контурных усилий прикладывается небольшая по величине равномерно распределённая поперечная нагрузка. Последующий расчёт производится для конических элементов без участия этой нагрузки. На рис. 4, а, б представлены эпюры нормальных перемещений $w$ и радиальных продольных усилий $N_{r}$, полученных для нагрузок $P$ различной интенсивности. В обозначениях использованы безразмерные параметры монографии [2]. Необходимо отметить, что независимо от метода расчёта наличие больших распорных усилий в сжато-изогнутой пластине существенно снижает сходимость итерационного процесса по сравнению со сходимостью «обычных» задач нелинейного деформирования. Приведенные графики получены при разбиении пластины по радиусу на $n=50$ равных участков и количестве итераций $i \leqslant 20$. При этом погрешность для перемещений и усилий, сравниваемых с аналогичными величинами, полученными методом последовательных приближений по параметру [2], не превышает 1 \%. На рис. 4, в представлены нормальные перемещений центра пластины в зависимости от различных значений $n$ и $P$. График, выделенный пунктирной линией, получен методом Бубнова-Галёркина с удержанием одного члена разложения $[7,8]$. Анализ результатов, полученных в центре пластины, показывает (рис. 4, г), что точность расчёта перемещений (кривая 1) и усилий (кривая 2) обеспечивается при различном количестве участков $n$.

\section{БИБЛИОГРАФИЧЕСКИЙ СПИСОК}

1. Кармишин А.В., Лясковеч В.А., Мяченков В.И., Фролов А.Н. Статика и динамика тонкостенных оболочечных конструкций. М.: Машиностроение, 1975. 376 с. [Karmishin A. V., Lyaskovets V.A., Myachenkov V.I., Frolov A. N. Statics and dynamics of thin-wall shell-type structures. Moscow: Mashinostroenie, 1975. 376 pp.]

2. Валишвили Н. В. Методы расчёта оболочек вращения на ЭЦВМ. М.: Машиностроение, 1976. 279 c. [Valishvili N. V. Methods of analyzing shells of revolution on a digital computer: Mashinostroenie, 1976. 279 pp.]

3. Timoshenko S. P., Woinowsky-Krieger S. Theory of Plates and Shells. New York: McGrawHill, 1959. 595 pp.; русск. пер.: Тимошенко С. П., Войновский-Кригер С. Пластины и оболочки. М.: Наука, 1966. 636 с.

4. Смирнов А.Ф., Александров А.В., Лащеников Б. Я., Шапошников Н. Н. Строительная механика. Тонкостенные пространственные системы / ред. А.Ф. Смирнов. М.: Стройиздат, 1983. 488 c. [Smirnov A.F., Aleksandrov A. V., Lashchenikov B. Ya., Shaposhnikov N. N. Civil engineering mechanics. Thin-walled spatial systems/ ed. A.F. Smirnov. Moscow: Stroyizdat, 1983. 488 pp.]

5. Еленицкий Э. Я. Расчёт свободных колебаний призматических систем с распределенными параметрами // Изв. вузов. Cmpouтельство, 1996. №7. С. 26-31. [Yelenitskiy E. Ya. The calculation of free oscillations for prismatic systems with distributed parameters // Izv. Vuzov. Stroitel'stvo, 1996. no. 7. Pp. 26-31].

6. Лукаш П. А. Основы нелинейной строительной механики. М.: Стройиздат, 1978. 204 с. 
[Lukash P. A. Basis of nonlinear civil engineering mechanics. Moscow: Stroyizdat, 1978. 204 pp.]

7. Вольмир А.С. Устойчивость деформируемых систем. М.: Наука, 1967. 984 с. [Vol'mir A. S. Stability of Deformable Systems. Moscow: Nauka, 1967. 984 pp.]

8. Григолюк Э.И. К вопросу о поведении круглой пластины после потери устойчивости // Вестник инженеров и техников, 1949. № 3. С. 103-106. [Grigoluk É. I. On the question of circular plate behavior after buckling // Vestnik Inzhenerov i Tekhnikov, 1949. no. 3. Pp. 103$106]$.

Поступила в редакцию $02 / \mathrm{V} / 2012$;

в окончательном варианте - 21/XI/2012.

MSC: 35Q74; 74K20, 74K25

BOUNDARY VALUE PROBLEM FOR THE FLEXIBLE AXIALLY

LOADED COMPOUND SHELLS OF REVOLUTION AND BEAMS SYSTEMS

\section{E. Ya. Elenitsky}

Samara State University of Architecture and Civil Engineering, 194, Molodogvardeyskaya st., Samara, 443001, Russia.

E-mail: elenit@list.ru

The effective algorithm of static calculation of geometrically nonlinear compound thin structures is offered. Linear differential equations of moment theory are used. Nonlinearity is considered by assigning unknown initial angular displacement of each segment retaining the form of the generating line. Unknown values of algebraic equations resolving system are the arbitrary constants of the general solution and the initial angles of generating lines rotation. Linearization is realized by Newton-Raphson iterative method and provides the high precision of results.

Key words: geometrical nonlinearity, beam, shell of revolution, angular deflection, differential equations, iterative calculation.

Original article submitted $02 / \mathrm{V} / 2012$;

revision submitted $21 / \mathrm{XI} / 2012$.

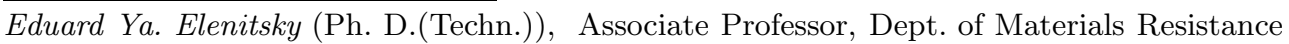
and Civil Engineering Mechanics. 